\title{
A Systematic Approach to Quality Oriented Product Sequencing for Multistage Manufacturing Systems
}

\author{
Faping Zhang ${ }^{1}$ and Shahid Ikramullah Butt ${ }^{2}$ \\ ${ }^{1}$ School of Mechanical Engineering, Beijing Institute of Technology, Beijing 100081, China \\ ${ }^{2}$ School of Mechanical and Manufacturing Engineering, National University of Sciences and Technology, Islamabad, Pakistan \\ Correspondence should be addressed to Faping Zhang; zfpnew@163.com
}

Received 1 June 2016; Revised 21 October 2016; Accepted 27 October 2016

Academic Editor: Thomas Hanne

Copyright (c) 2016 F. Zhang and S. I. Butt. This is an open access article distributed under the Creative Commons Attribution License, which permits unrestricted use, distribution, and reproduction in any medium, provided the original work is properly cited.

Product sequencing is one way to reduce cost and improve product quality for multistage manufacturing systems (MMS). However, systematically evaluating the influence of product sequence on quality performance for MMS is still a challenge. By considering the rate of incoming conforming product, manufacturing system quality transition between batch to batch, and quality propagation along stages, this paper investigates the appropriate batch policies and product sequencing for MMS so that satisfied quality performance can be achieved. A model to analyze the relationship between the product sequencing and quality performance is conducted just by using the quality inspection data and the complex engineering knowledge used in the variation method is avoided. Based on Markov Chain processes methodology, quality performance is modeled as a function of transition states jointly determined by multistage condition, product sequencing, incoming part quality, and propagation of the rate of conforming products among multistage. Quality related batch strategies are discussed for optimal quality performance. Two kinds of quality efficiency are put forward to facilitate the modeling and the discussion. The results of the model will lead to guidelines for quality management in multistage manufacturing systems.

\section{Introduction}

(A) Motivation. The quality performance of a multistage manufacturing system reflects the capability of the system delivering conforming quality features during manufacturing (i.e., to meet process specification) at each stage. However, the product quality is determined not only by the stability of the manufacturing processes, but also by the layout of process and product sequencing. It is desirable to conduct a systematic research that addresses quality assurance in all phases of product realization in terms of rate of confirming parts, which is the result of daily production quality inspection.

Production sequence and batch size are the components of production operation mostly concerned in production scheduling. Improvement in these components is one way to reduce cost and also improve product quality for multistage manufacturing systems (MMS). For instance, during machining processes, fixtures are used to locate workpiece in a given location. Uncertainty would increase when adjusting fixture locator in bidirection than always in one direction during batch to batch production, which is affected by the change of product types from batch to batch. Hence different process variability and risk of product quality are introduced during production operation. Furthermore, for multistage machining processes, between the machining stages, there may be different adjustment requirements for fixtures at each stage, which impact the success of the fixtures adjustment and the product quality transition during the batch production transitions. Another example of the case is the double color (or multiple color) car painting process, where at least two painting stages will be used (depending on how colorful a car is). Each stage may have different color painting sequence according to production operation between the batch to batch changeovers. As we know, at each stage, the paint quality is strongly correlated to the number of available paint colors [1] and it may temporarily fluctuate with color change [2] and propagate to next stage.

Although product quality is one of important issues in MMS due to changeover, the impact of production sequencing and batch policies on quality for a multistage 
manufacturing system with batch production has not been addressed. It is still a challenge to evaluate quality performance with production operation for MMS without the intensive engineering knowledge. On the other hand, various types of readily available data create tremendous opportunities for developing an evaluation methodology. For example, the first part of a batch will be totally inspected to ensure the successful transition during product changeover. Hence, this data can be used to evaluate transition concerning the product quality. This paper investigates the appropriate batch size and production sequence, so that the quality requirement is satisfied.

The remainder of the paper is organized as follows: in Section 1(B), a brief review of relevant literature is presented. In Sections 2 and 3, the problem statement is discussed in detail and quality performance modeling is formulated. Section 4 provides intensively evaluation of the MMS quality performance. In Section 5 an example and discussion are given. The conclusions are drawn in Section 6.

(B) Literature Review. The evaluation of the performance of manufacturing system's quality has been focused as an important research issue in quality control area. It has been approached from two perspectives. The first is to study the variation propagation along stages by means of the intensive usage of engineering knowledge for multistage manufacturing system. Lots of researchers have developed the methodology of Stream of Variation (SoV) to model the multistage variation accumulation [3-8] for machining and assembly process and the state space model is one of the frequently used tools. The SoV based quality modeling not only emphasizes on describing the forming of variation along the stages but also curbs the variation. Hence, intensive engineering knowledge is involved. Moreover, not enough research is carried out to study the quality issues related to the rate of incoming and outgoing conforming product with systematic approach, which is the actual requirement in quality performance evaluation of manufacturing system while there is abundance of historical production data which has not been given much attention previously.

Secondly, full utilization of the measured data during manufacturing process inspection for quality control and then evaluating the quality performance of manufacturing system has also been studied. The measured data is usually used to quantify the rate of nonconformance of the outgoing units of the manufacturing system. The attribute control chart used for the statistical process control of the manufacturing process is one of the traditional approaches in this regard. To evaluate the quality performance of the manufacturing system with different setting, series of studies have been done [9-14]. Quality parameters of machines in serial production lines have been analyzed to trade off the machine efficiency $[9,10]$. A revised learning model with time-varying learning rates was proposed for embedding learning effects into a multistage assembly/production setting for suppliers with the rate of nonconformance units along with the inspection sample rates [11]. Learning curve was also used to reduce the percentage of defective items per lot produced by manufacturing system with 100 per cent screening [12]. The above research is mainly concerned about the setting up of the machining system. The impact of manufacturing system configuration including the inspection policies, buffer dimensioning on the performance of the system in terms of reliability and productivity, product quality, capacity, scalability, and cost has also been discussed by some researchers [13-15].

Actually, the input factors, such as the product sequencing and batching of the manufacturing system, also have a significant influence on the system quality performance. Being one of the most effective ways to improve production system efficiency, product sequencing has been studied extensively in the field of production scheduling. The scheduling problem considers a batch capacity constraint, sequence-dependent processing times, incompatible product families, additional resources, machine capability, and product sequence to achieve scheduling aim $[16,17]$. Quality issue has not been considered much as constraint factor or the scheduling aim, although few researchers have combined quality issue with production scheduling $[18,19]$ for a special kind of the production process only. Series of researches $[20,21]$ have been conducted and a quantitative model based on Markov Chain to evaluate the quality performance of a flexible machining system was presented which is considered as a single manufacturing system. The work is mainly focused on the state transition properties of the single-stage between batch to batch operations. The research is primarily focused on one single-stage manufacturing system without incoming part quality. So this gives us an opportunity to conduct a thorough study on quality performance evaluation for MMS under different production operation.

With the emergence of new manufacturing systems such as reconfiguration system with the dynamic and highly competitive marketplace demands, evaluation of quality performance from multistage aspect is becoming more and more crucial. Another aspect is to evaluate it from the system level to predict quality performance according to the rate of incoming conforming product to that of outgoing product in a statistical way, where the detailed engineering knowledge along the multistage manufacturing system is not concerned.

\section{Problem Formulation}

For a given multistage manufacturing system producing variety of products, the following assumptions are used to address the issues of the manufacturing system, product types, production sequence, and product quality.

(1) The multistage manufacturing system has $m$ serial stages and processes $n$ different types of products. Each product type $i$ is manufactured in a batch with batch size $b_{i}, b_{i} \geq 1$ and $i=1,2, \ldots, n$.

(2) For $n$ different types of parts to be processed in a manufacturing system, there are $(n-1)$ ! kinds of product sequences, denoted as $s^{l}, l=1, \ldots,(n-1)$ !. The products are introduced into the system with sequence $s^{l}=\left\{s_{1}^{l}, s_{2}^{l}, \ldots, s_{n}^{l}\right\}$, where $s_{t}^{l}$ denotes the $t$ th product type in the sequence $s^{l}$, and $t \in\{1,2, \ldots, n\}$, $s_{t}^{l} \in\{1,2, \ldots, n\}$. 
(3) Every product enters the manufacturing system with a certain rate of conforming product $\gamma_{s_{t}^{l}, j}$ which will change along the stages.

(4) For each sequence $s^{l}$, the multistage manufacturing system will work on product type $s_{t}^{l}$ for $b_{s_{t}^{l}}$ parts before switching to product type $s_{t+1}^{l}$. It is assumed that product type $s_{1}^{l}$ is processed again after processing type $s_{n}^{l}$.

(5) The $k$ th stage state of a multistage manufacturing system is in normal state $o_{s_{i}^{l}, j, k}$ or abnormal state $f_{s_{i}^{l}, j, k}$, $i=1, \ldots, n, j=1, \ldots, b_{s_{i}^{l}}$, when it is processing the $j$ th part in the batch of the product type $s_{i}^{l}$ with good quality or with defects, respectively.

(6) The output part quality of the $k$ th stage of a multistage manufacturing system is in good state $g_{s_{i}, j, k}$ or defective state $d_{s_{i}^{l}, j, k}, i=1, \ldots, n, j=1, \ldots, b_{s_{i}^{l}}$, if it is processing the $j$ th part in the batch of the product type $s_{i}^{l}$ with good quality or with defects, respectively. Thus, there are $2 B, B=\sum_{i=1}^{n} b_{i}$, states in the system for a given sequence, defined by the quality status, product type processed, and its position within a batch.

(7) When the $k$ th stage is in normal state $o_{s_{i}^{l}, j, k}, s_{i}^{l}=$ $1, \ldots, n, j=1, \ldots, b_{s_{i}}-1, k=1, \ldots, m$, it has probabilities $\lambda_{s_{i}^{l}, s_{i}^{l}, k}$ to transit to abnormal state $f_{s_{i}^{l}, j+1, k}$ and $1-\lambda_{s_{i}^{l}, s_{i}^{l}, k}$ to normal state $o_{s_{i}^{l}, j+1, k}$. Similarly, when the stage is in abnormal state $f_{s_{i}, j, k}^{l}, s_{i}^{l}=1, \ldots, n$, $j=1, \ldots, b_{s_{i}^{l}}-1, k=1, \ldots, m$, it can transit to normal state $o_{s_{i}^{l}, j+1, k}$ with probability $\mu_{s_{i}^{l}, s_{i}^{l}, k}$ and to abnormal state $f_{s_{i}^{l}, j+1, k}$ with $1-\mu_{s_{i}^{l}, s_{i}^{l}, k}$ probability.

(8) When the $k$ th stage is processing the last part within a batch and in normal state $o_{s_{i}^{l}, b_{l_{i}}, k}, s_{i}^{l}=1, \ldots, n, j=$ $1, \ldots, b_{s_{i}^{l}}-1, k=1, \ldots, m$, it has probabilities $\lambda_{s_{i+1}^{l}, s_{i}^{l}, k}$ and $1-\lambda_{s_{i+1}^{l}, s_{i}^{l}, k}$ to transit to states $f_{s_{i+1}^{l}, 1, k}$ and $o_{s_{i+1}^{l}, 1, k}^{l}$, respectively. Similarly, when the stage is in abnormal stage $f_{s_{i}^{l}, b_{s_{i}}, k}$, it has probabilities $\mu_{s_{i+1}^{i}, s_{i}^{l}, k}$ and $1-\mu_{s_{i+1}^{l}, s_{i}^{l}, k}$ to transit to states $o_{s_{i+1}^{l}, 1, k}$ and $f_{s_{i+1}^{l}, 1, k}$, respectively.

(9) When the $k$ th stage is in state $o_{s_{n}}, b_{s_{n}}, k$, it has probabilities $\lambda_{s_{1}^{l}, s_{n}^{l}, k}$ and $1-\lambda_{s_{1}^{l}, s_{n}^{l}, k}$ to transit to states $f_{s_{1}^{l}, 1, k}$ and $o_{s_{1}^{l}, 1, k}$, respectively. Similarly, when the $k$ th stage is in state $f_{s_{n}^{l}, b_{s_{n}}, k}$, it has probabilities $\mu_{s_{1}^{l}, l_{n}^{l}, k}$ and $1-\mu_{s_{1}^{l}, s_{n}^{l}, k}$ to transit to states $o_{s_{1}^{l}, 1, k}$ and $f_{s_{1}^{l}, 1, k}$, respectively. It is sure that all $0<\lambda_{i j}<1,0<\mu_{i j}<1, \forall i, j$.

(10) Another assumption including the inspection policy is one hundred percent inspection which is error-free.

Let $P\left(o_{s_{i}^{l}, j, k}\right)$ and $P\left(f_{s_{i}^{l}, j, k}\right), s_{i}^{l}=1, \ldots, n, j=1, \ldots, b_{s_{i}^{l}}-1$, $k=1, \ldots, m$, denote the probabilities that the $k$ th stage is in state $o_{s_{i}^{l}, j, k}$ or $f_{s_{i}^{l}, j, k}$, respectively. $P\left(g_{s_{i}^{l}, j, k}\right)$ and $P\left(d_{s_{i}^{l}, j, k}\right)$, $s_{i}^{l}=1, \ldots, n, j=1, \ldots, b_{s_{i}^{l}}-1, k=1, \ldots, m$, denote the

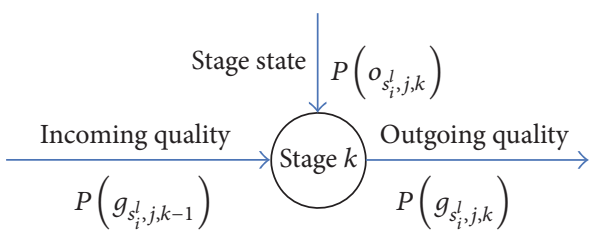

FIGURE 1: Schematic of single-stage qualities.

probabilities that the $k$ th stage is to output part quality state in $g_{s_{i}^{l}, j, k}$ and $d_{s_{i}^{l}, j, k}$ respectively. So $P\left(g_{s_{i}^{l}, j, m}\right)$ and $P\left(d_{s^{l}, j, m}\right)$ should be the probabilities of the system's output part quality state: good or defective, which can also be denoted as $P\left(g_{s_{i}^{l}, j}\right)$ and $P\left(d_{s_{i}^{l}, j}\right)$. Thus,

$$
\begin{aligned}
& P\left(g_{s_{i}^{l}, j}\right)=P\left(g_{s_{i}^{l}, j, m}\right), \\
& P\left(d_{s_{i}^{l}, j}\right)=P\left(d_{s_{i}^{l}, j, m}\right) .
\end{aligned}
$$

Then the overall quality performance of a multiple stage manufacturing system for a given sequence $s_{i}^{l}$, that is, the probability to produce a good or a defective part in batch production, is defined as $P\left(g_{b t}^{l}\right)$ and $P\left(d_{b t}^{l}\right)$, respectively and expressed mathematically as

$$
P\left(g_{b t}^{l}\right)=\sum_{s_{i}^{l}=1}^{n} \sum_{j=1}^{b_{s_{i}}} P\left(g_{s_{i}^{l}, j}\right) P\left(d_{b t}^{l}\right)=\sum_{s_{i}^{l}=1}^{n} \sum_{j=1}^{b_{s_{i}^{l}}} P\left(d_{s_{i}^{l}, j}\right) .
$$

\section{Quality Performance Modeling}

Literature [22] represents two categories influencing factors of the output part quality of a multistage manufacturing system, that is, incoming part quality and manufacturing system conditions related to output quality such as the machine reliability. The Bernoulli quality model can be used to consider both conditions [23]. We can employ Bernoulli quality model to establish the relationship among incoming part quality, manufacturing system conditions, and outgoing product quality, from which we can also fulfill the quality performance evaluation of the multistage manufacturing system.

3.1. General Modeling. Consider a serial, multistage manufacturing process producing one type of product at a time. At each stage there are two factors contributing to the output quality of the machining stage, namely, incoming part quality and the stage transition rate as shown in Figure 1.

So for the $k$ th stage, we have

$$
\begin{aligned}
P\left(g_{s_{i}^{l}, j, k}\right)= & P\left(g_{s_{i}^{l}, j, k-1}\right) \cdot P\left(o_{s_{i}^{l}, j, k}\right), \\
P\left(d_{s_{i}^{l}, j, k}\right)= & P\left(g_{s_{i}^{l}, j, k-1}\right) \cdot P\left(f_{s_{i}^{l}, j, k}\right)+P\left(d_{s_{i}^{l}, j, k-1}\right) \\
& \cdot P\left(o_{s_{i}^{l}, j, k}\right)+P\left(d_{s_{i}^{l}, j, k-1}\right) \cdot P\left(f_{s_{i}^{l}, j, k}\right) .
\end{aligned}
$$




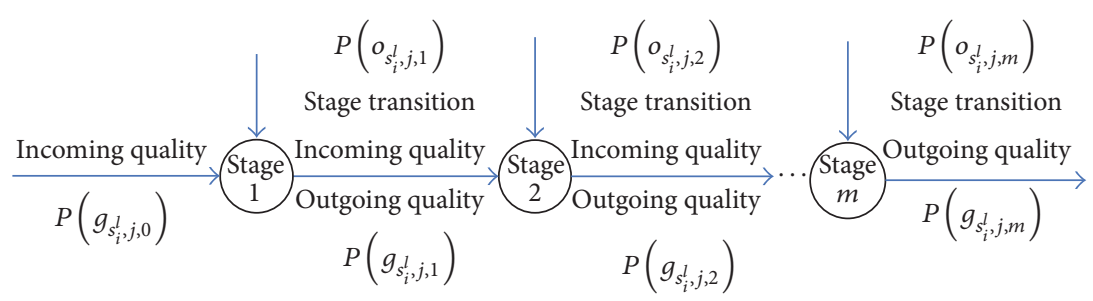

FIGURE 2: Schematic of multistage qualities.

Analogously, for the $j-1$ product in batch $s_{i}^{l}$ at the $k$ th stage, we have

$$
\begin{aligned}
P\left(g_{s_{i}^{l}, j-1, k}\right)= & P\left(g_{s_{i}^{l}, j-1, k-1}\right) \cdot P\left(o_{s_{i}^{l}, j-1, k}\right), \\
P\left(d_{s_{i}^{l}, j-1, k}\right)= & P\left(g_{s_{i}^{l}, j-1, k-1}\right) \cdot P\left(f_{s_{i}^{l}, j-1, k}\right) \\
& +P\left(d_{s_{i}^{l}, j-1, k-1}\right) \cdot P\left(o_{s_{i}^{l}, j-1, k}\right) \\
& +P\left(d_{s_{i}^{l}, j-1, k-1}\right) \cdot P\left(f_{s_{i}^{l}, j-1, k}\right) .
\end{aligned}
$$

From the assumptions (1)-(9), when $1<j \leq b_{s_{i}^{l}}$, that is, transition occurs within the batch without change product type, we have

$$
\begin{aligned}
P\left(o_{s_{i}^{l}, j, k}\right)= & P\left(o_{s_{i}^{l}, j-1, k}\right) \cdot\left(1-\lambda_{s_{i}^{l}, s_{i}^{l}, k}\right)+P\left(f_{s_{i}^{l}, j-1, k}\right) \\
& \cdot \mu_{s_{i}^{l}, s_{i}^{l}, k}, \\
P\left(f_{s_{i}^{l}, j, k}\right)= & P\left(o_{s_{i}^{l}, j-1, k}\right) \cdot \lambda_{s_{i}^{l}, s_{i}^{l}, k}+P\left(f_{s_{i}^{l}, j-1, k}\right) \\
& \cdot\left(1-\mu_{s_{i}^{l}, s_{i}^{l}, k}\right) .
\end{aligned}
$$

Then combining (3) to (8), we have

$$
\begin{aligned}
P\left(g_{s_{i}^{l}, j, k}\right) & \\
= & P\left(g_{s_{i}^{l}, j, k-1}\right) \\
& \cdot\left[e_{s_{i}^{l}, j-1, k}\left(1-\lambda_{s_{i}^{l}, s_{i}^{l}, k}\right)+\left(1-e_{s_{i}^{l}, j-1, k}\right) \mu_{s_{i}^{l}, s_{i}^{l}, k}\right] .
\end{aligned}
$$

For the detailed conduction of (9), see Appendix A, where $e_{s_{i}^{l}, j-1, k}=P\left(g_{s_{i}^{l}, j-1, k}\right) / P\left(g_{s_{i}^{l}, j-1, k-1}\right)$ denotes the stage to stage quality efficiency of the $k$ th stage at processing $s_{i}^{l}$ batch and $j-1$ part. So we can get

$$
e_{s_{i}^{l}, j, k}=e_{s_{i}^{l}, j-1, k}\left(1-\lambda_{s_{i}^{l}, s_{i}^{l}, k}\right)+\left(1-e_{s_{i}^{l}, j-1, k}\right) \mu_{s_{i}^{l}, s_{i}^{l}, k} .
$$

As Figure 2 shows the whole manufacturing system outgoing product quality; namely, the outgoing quality of the system for the sequence $s_{i}^{l}$ of $j$ th part should be

$$
\begin{aligned}
& P\left(g_{s_{i}^{l}, j}\right)=P\left(g_{s_{i}^{l}, j, m}\right)=\frac{1}{\sum_{i=1}^{n} b_{s_{i}^{l}}} \\
& \cdot \prod_{k=1}^{m}\left[e_{s_{i}^{l}, j-1, k}\left(1-\lambda_{s_{i}^{l}, s_{i}^{l}, k}\right)+\left(1-e_{s_{i}^{l}, j-1, k}\right) \mu_{s_{i}^{l}, s_{i}^{l}, k}\right] \\
& \cdot \gamma_{s_{i}^{l}}=\frac{1}{\sum_{i=1}^{n} b_{s_{i}^{l}}} \prod_{k=1}^{m} e_{s_{i}^{l}, j, k} \cdot \gamma_{s_{i}^{l}}=\frac{1}{\sum_{i=1}^{n} b_{s_{i}^{l}}} e_{s_{i}^{l}, j} \cdot \gamma_{s_{i}^{l}},
\end{aligned}
$$

where $e_{s_{i}^{l}, j}=\prod_{k=1}^{m} e_{s_{i}^{l}, j, k}$ is the manufacturing system's stage to stage quality efficiency for $j$ th part in the production sequence $s_{i}^{l}$ and $P\left(g_{s_{i}^{l}, j, m}\right)$ is outgoing quality of the final stage of the system for the sequence $s_{i}^{l}$.

Similarly, when $j=1$ and $i>1$, transition is from the last part in previous batch to the first part in current batch. A product switch from type $s_{i-1}^{l}$ to type $s_{i}^{l}$ is involved. Hence, we can get

$$
P\left(g_{s_{i}^{l}, 1}\right)=P\left(g_{s_{i}^{l}, 1, m}\right)=\frac{1}{\sum_{i=1}^{n} b_{s_{i}^{l}}} e_{s_{i}^{l}, 1} \cdot \gamma_{s_{i}^{l}},
$$

where $e_{s_{i}^{l}, 1}=\prod_{k=1}^{m}\left[e_{s_{i}^{l}, b_{s_{i-1}}, k}\left(1-\lambda_{s_{i}^{l}, s_{i-1}^{l}, k}\right)+\left(1-e_{s_{i}^{l}, b_{s_{i-1}}, k}\right) \mu_{s_{i}^{l}, s_{i-1}^{l}, k}\right]$. Finally, if $i=j=1$, a product switch from type $s_{n}^{l}$ to type $s_{1}^{l}$ occurs. Thus, it follows that

$$
P\left(g_{s_{1}^{l}, 1}\right)=P\left(g_{s_{1}^{l}, 1, m}\right)=\frac{1}{\sum_{i=1}^{n} b_{s_{i}^{l}}} e_{s_{1}^{l}, 1} \cdot \gamma_{s_{1}^{l}},
$$

where $e_{s_{1}^{l}, 1}=\prod_{k=1}^{m}\left[e_{s_{1}^{l}, b_{s_{n}}, k}\left(1-\lambda_{s_{1}^{l}, s_{n}^{l}, k}\right)+\left(1-e_{s_{1}^{l}, b_{s_{n}^{l}}, k}\right) \mu_{s_{1}^{l}, s_{n}^{l}, k}\right]$. So, the product quality is denoted as $P\left(g_{s_{i}^{l}, j}\right)$;

$$
\begin{aligned}
& P\left(g_{b t}^{l}\right)=\sum_{i=1}^{n} \sum_{j=1}^{b_{s_{i}^{l}}} P\left(g_{s_{i}^{l}, j}\right)=\frac{1}{\sum_{i=1}^{n} b_{s_{i}^{l}}} \sum_{i=1}^{n} \sum_{j=1}^{b_{s_{i}^{l}}} e_{s_{i}^{l}, j} \cdot \gamma_{s_{i}^{l}} \\
& \quad=\frac{1}{\sum_{i=1}^{n} b_{s_{i}^{l}}}\left(\sum_{i=1}^{n}\left(b_{s_{i}^{l}}-1\right) e_{s_{i}^{l}, 2} \cdot \gamma_{s_{i}^{l}}+\sum_{i=2}^{n} e_{s_{i}^{l}, 1} \cdot \gamma_{s_{i}^{l}}\right. \\
& \left.+e_{s_{1}^{l}, 1} \cdot \gamma_{s_{1}^{l}}\right) .
\end{aligned}
$$

In addition, the total probability is equal to 1 :

$$
\sum_{i=1}^{n} \sum_{j=1}^{b_{s_{i}}}\left(P\left(g_{s_{i}^{l}, j}\right)+P\left(d_{s_{i}^{l}, j}\right)\right)=1 .
$$

We assume that

$$
\begin{aligned}
\delta_{s_{i}^{l}, s_{i}^{l}, k}= & 1-\lambda_{s_{i}^{l}, s_{i}^{l}, k}-\mu_{s_{i}^{l}, s_{i}^{l}, k} \\
& s_{i}^{l}=1, \ldots, n, \quad j=1, \ldots, b_{s_{i}^{l}}-1, k=1, \ldots, m .
\end{aligned}
$$


So (10) can be rewritten as

$$
\begin{aligned}
e_{s_{i}^{l}, j, k} & =e_{s_{i}^{l}, j-1, k}\left(1-\lambda_{s_{i}^{l}, s_{i}^{l}, k}\right)+\left(1-e_{s_{i}^{l}, j-1, k}\right) \mu_{s_{i}^{l}, s_{i}^{l}, k} \\
& =\delta_{s_{i}^{l}, s_{i}^{l}, k} e_{s_{i}^{l}, j-1, k}+\mu_{s_{i}^{l}, s_{i}^{l}, k} .
\end{aligned}
$$

Similarly,

$$
\begin{aligned}
e_{s_{i}^{l}, 1, k}= & e_{s_{i-1}^{l}, b_{s_{i-1}^{l}}, k}\left(1-\lambda_{s_{i}^{l}, s_{i-1}^{l}, k}\right) \\
& +\left(1-e_{s_{i-1}^{l}, b_{s_{i-1}^{l}}, k}\right) \mu_{s_{i}^{l}, s_{i-1}^{l}, k} \\
= & \delta_{s_{i}^{l}, s_{i-1}^{l}, k} e_{s_{i-1}^{l}, b_{s_{i-1}^{l}}, k}+\mu_{s_{i}^{l}, s_{i-1}^{l}, k}, \\
e_{s_{1}^{l}, 1, k}= & e_{s_{n}^{l}, b_{s_{n}^{l}}, k}\left(1-\lambda_{s_{1}^{l}, s_{n}^{l}, k}\right)+\left(1-e_{s_{n}^{l}, b_{s_{n}^{l}}, k}\right) \mu_{s_{1}^{l}, s_{n}^{l}, k} \\
= & \delta_{s_{1}^{l}, s_{n}^{l}, k} e_{s_{n}^{l}, b_{s_{n}^{l}}, k}+\mu_{s_{1}^{l}, s_{n}^{l}, k} .
\end{aligned}
$$

Vector $E_{k}, \Phi_{k}$, and matrix $\Psi_{k}$ are introduced as follows:

$$
\begin{aligned}
& E_{k}=\left[e_{s_{1}^{l}, 1, k}, \ldots, e_{s_{1}^{l}, b_{s_{1}}, k}, e_{s_{2}^{l}, 1, k}, \ldots, e_{s_{2}^{l}, b_{s_{2}}, k}, \ldots, e_{s_{n}^{l}, 1, k}, \ldots, e_{s_{n}^{l}, b_{s_{n}}, k}\right]^{\mathrm{T}}, \\
& \Phi_{k}=\left[\mu_{s_{1}^{l}, s_{n}^{l}, k}, \mu_{s_{1}^{l}, s_{1}^{l}, k}, \ldots, \mu_{s_{1}^{l}, s_{1}^{l}, k}, \mu_{s_{2}^{l}, s_{1}^{l}, k}, \mu_{s_{2}^{l}, s_{2}^{l}, k}, \ldots, \mu_{s_{n}^{l}, s_{n-1}^{l}, k},\right. \\
& \left.\mu_{s_{n}^{l}, s_{n}^{l}, k}, \ldots, \mu_{s_{n}^{l}, s_{n}^{l}, k}\right]^{\mathrm{T}},
\end{aligned}
$$

$\Psi_{k}$

$$
=\left[\begin{array}{cccccccc}
0 & \cdots & 0 & 0 & 0 & \cdots & 0 & \delta_{s_{1}^{l}, s_{n}^{l}, k} \\
\delta_{s_{1}^{l}, s_{1}^{l}, k} & \cdots & 0 & 0 & 0 & \cdots & 0 & 0 \\
0 & \ddots & 0 & 0 & 0 & \cdots & 0 & 0 \\
0 & \cdots & \delta_{s_{1}^{l}, s_{1}^{l}, k} & 0 & 0 & \cdots & 0 & 0 \\
0 & \cdots & 0 & \delta_{s_{2}, s_{1}^{l}, k} & 0 & \cdots & 0 & 0 \\
0 & \cdots & 0 & 0 & \delta_{s_{2}, s_{2}^{l}, k} & \cdots & 0 & 0 \\
0 & \cdots & 0 & 0 & 0 & \ddots & 0 & 0 \\
0 & \cdots & 0 & 0 & 0 & \cdots & \delta_{s_{n}^{l}, s_{n}^{l}, k} & 0
\end{array}\right] .
$$

So (16), (17), and (18) can be rewritten as

$$
E_{k}=\Psi_{k} E_{k}+\Phi_{k}
$$

It follows that

$$
E_{k}=\left(\mathrm{I}-\Psi_{k}\right)^{-1} \Phi_{k}
$$

Note that the inverse always exists due to the fact that an irreducible Markov Chain with finite number of states has a unique solution. Equation (19) can be used to calculate stage quality efficiency at each stage. We can reorganize the $E_{k}$ as diagonal matrix as follows:

$$
\begin{aligned}
E_{k} & =\left[\begin{array}{cccc}
e_{s_{1}^{l}, 1, k} & 0 & 0 & 0 \\
0 & e_{s_{1}^{l}, 2, k} & 0 & 0 \\
0 & 0 & \ddots & 0 \\
0 & 0 & 0 & e_{s_{n}^{l}, b_{s_{n}^{l}}, k}
\end{array}\right], \\
E_{k-1} & =\left[\begin{array}{cccc}
e_{s_{1}^{l}, 1, k-1} & 0 & 0 & 0 \\
0 & e_{s_{1}^{l}, 2, k-1} & 0 & 0 \\
0 & 0 & \ddots & 0 \\
0 & 0 & 0 & e_{s_{n}^{l}, b_{s_{n}^{l}}, k-1}
\end{array}\right] .
\end{aligned}
$$

The rate of incoming conforming quality of each part is denoted as

$$
\Gamma_{s_{i}^{l}}=\left[\begin{array}{cccc}
\gamma_{s_{1}^{l}, 1} & 0 & 0 & 0 \\
0 & \gamma_{s_{1}^{l}, 2} & 0 & 0 \\
0 & 0 & \ddots & 0 \\
0 & 0 & 0 & \gamma_{s_{n}^{l}, b_{s_{n}}}
\end{array}\right] .
$$

Introduce vectors $X_{k}$ as follows to represent the good part probability of the $k$ th stage.

$$
\begin{gathered}
X_{k}=\left[P\left(g_{s_{1}^{l}, 1, k}\right), \ldots, P\left(g_{s_{1}^{l}, b_{s_{1}^{l}}, k}\right), P\left(g_{s_{2}^{l}, 1, k}\right), \ldots,\right. \\
\left.P\left(g_{s_{2}^{l}, b_{s_{2}^{l}}, k}\right), \ldots, P\left(g_{s_{n}^{l}, 1, k}\right), \ldots, P\left(g_{s_{n}^{l}, b_{s_{n}}, k}\right)\right]^{\mathrm{T}} .
\end{gathered}
$$

$$
\text { So } X_{k}=\left(1 / \sum_{i=1}^{n} b_{s_{i}^{l}}\right) E_{k} \Gamma_{s_{i}^{l}}=\left(1 / \sum_{i=1}^{n} b_{s_{i}^{l}}\right)\left(I-\Psi_{k}\right)^{-1} \Phi_{k} \Gamma_{s_{i}^{l}} \text {. }
$$

The final probability of good parts should be equal to the outgoing good parts of the last $m$ th stage:

$$
X=\frac{1}{\sum_{i=1}^{n} b_{s_{i}^{l}}} \prod_{k=1}^{m} E_{k} \Gamma_{s_{i}^{l}}=\frac{1}{\sum_{i=1}^{n} b_{s_{i}^{l}}} \prod_{k=1}^{m}\left(I-\Psi_{k}\right)^{-1} \Phi_{k} \Gamma_{s_{i}^{l}} .
$$

Therefore, the probability of good parts $P\left(g_{b t}^{l}\right)$ is evaluated as follows.

Theorem 1. Under assumptions (1)-(9), the probability of good parts $P\left(g_{b t}^{l}\right)$ is calculated by

$$
P\left(g_{b t}^{l}\right)=\sum_{i=1}^{B} x_{i}
$$


where $B=\sum_{i=1}^{n} b_{i}$ and $x_{i}$ is element of $X$ and can be solved from (21) and thus

$$
\begin{aligned}
P\left(g_{b t}^{l}\right)= & \frac{\sum_{i=1}^{n}\left(b_{s_{i}^{l}}-1\right) \prod_{k=1}^{m}\left(\delta_{s_{i}^{l}, s_{i}^{l}, k} e_{s_{i}^{l}, j-1, k}+\mu_{s_{i}^{l}, s_{i}^{l}, k}\right) \cdot \gamma_{s_{i}^{l}}}{\sum_{i=1}^{n} b_{s_{i}^{l}}} \\
& +\frac{\sum_{i=2}^{n} \prod_{k=1}^{m}\left(\delta_{s_{i}^{l}, s_{i-1}^{l}, k} e_{s_{i-1}^{l}, b_{s_{i-1}}, k}+\mu_{s_{i}^{l}, s_{i-1}^{l}, k}\right) \cdot \gamma_{s_{i}^{l}}+\prod_{k=1}^{m}\left(\delta_{s_{1}^{l}, s_{n}^{l}, k} e_{s_{n}^{l}, b_{s_{n}^{l}}, k}+\mu_{s_{1}^{l}, s_{n}^{l}, k}\right) \cdot \gamma_{s_{1}^{l}}}{\sum_{i=1}^{n} b_{s_{i}^{l}}} .
\end{aligned}
$$

Many manufacturing plans accumulate a lot of production data including all transition probabilities. Then, using these probabilities, Theorem 1 can be used to evaluate the product quality.

\section{Quality Oriented Product Sequencing}

Product sequencing does impact on quality performance of manufacturing systems. Some researchers have addressed this issue for individual stage production [24]. They approached this issue for car body painting of color changeover [25]. They assumed that these works imply that color changes do impact paint quality but their work is mainly focused on the individual stage production.

For multistage manufacturing systems research is not conducted in the same way. Actually it is also true that product sequencing has a certain impact on quality performance of multistage manufacturing system.

4.1. Optimal Product Sequences for General Cases. For product sequencing, we want to know which sequence will have the best quality performance under the known transition data and the rate of incoming conforming product. From the above assumptions, we have the following corollary.

Corollary 2. Under assumptions (1)-(9), the optimal production sequence is the sequence that satisfies

$$
\min _{S_{i}^{l}} \sum_{i=1}^{n} \sum_{j=1}^{b_{s_{i}^{l}}}\left|e_{s_{i}^{l}, j}-\gamma_{s_{i}}\right| .
$$

It is easy to prove that if two pieces of data have identical sum, then the smaller the range of the two data, the bigger the multiple of the two data. We also know that the optimal product sequence is the sequence to make the $P\left(g_{b t}^{l}\right)$ largest. Namely, the bigger the rate of incoming product conformance $\gamma_{s_{i}^{l}}$ is, the larger the corresponding system quality transition $e_{s_{i}^{l}, j}=\prod_{k=1}^{m}\left[e_{j-1, k}\left(1-\lambda_{s_{i}^{l}, s_{i}^{l}, k}\right)+\left(1-e_{j-1, k}\right) \mu_{s_{i}^{l}, s_{i}^{l}, k}\right]$ is and both of them should have the same sequence, namely, for small to large or from large to small.

It is easy to see that the transition probabilities within batches do not play a role in searching for optimal value because the value of $\left|e_{s_{i}^{l}, j}-\gamma_{s_{i}^{l}}\right|$ is identical in one batch for $j \neq 1$; thus only every $\left|e_{s_{i}^{l}, 1}-\gamma_{s_{i}^{l}}\right|$ is different. Hence, we have the following corollary.

Corollary 3. Under assumptions (1)-(9), the optimal production sequence is the sequence that satisfies

$$
\min _{S_{i}^{l}} \sum_{i=1}^{n}\left|e_{s_{i}^{l}, 1}-\gamma_{s_{i}^{l}}\right| .
$$

4.2. Sequence and Quality Efficiency. Quality efficiency, a very important quality performance index for multistage manufacturing system, is not only determined by system properties but also affected by the products sequence. Although quality efficiency has been studied intensively but not in multistage manufacturing system and at the same time the effect on sequencing is not considered [20]. The aim of this section is to investigate the relationship between sequence and quality efficiency.

Definition 4 (batch to batch quality transition efficiency). At the $k$ th stage, it is defined as

$$
\begin{aligned}
T_{s_{i}^{l}, s_{i}^{l}, k}= & \frac{\mu_{s_{i}^{l}, s_{i}^{l}, k}}{\lambda_{s_{i}^{l}, s_{i}^{l}, k}+\mu_{s_{i}^{l}, s_{i}^{l}, k}} \\
& s_{i}^{l}=1, \ldots, n, \quad j=1, \ldots, b_{s_{i}^{l}}-1, k=1, \ldots, m .
\end{aligned}
$$

Corollary 5. Under assumptions (1)-(9), the following statement holds

$$
e_{s_{i}^{l}, j, k}>e_{s_{i}^{l}, j-1, k} \quad \text { iff } T_{s_{i}^{l}, s_{i}^{l}, k}>e_{s_{i}^{l}, j-1, k} .
$$

Proof. See Appendix B.

This corollary indicates that if we know the former part's quality efficiency at the stage and the stage quality transition efficiency between batch to batch is higher than that, then the outgoing part quality efficiency should be larger than the former part quality efficiency at this stage. This can be used to predict product quality at the $k$ th stage if we know the former batch quality efficiency at the $k$ th stage and the stage quality transition efficiency. 
TABLE 1: The color of the three double color cars.

\begin{tabular}{lccc}
\hline Colors & Car 1 & Car 2 & Car 3 \\
\hline Main color & White & Black & Gray \\
Minor color & Gray & White & Black \\
\hline
\end{tabular}

TABLE 2: The transition parameters.

\begin{tabular}{lccccc}
\hline \multicolumn{2}{c}{ Transition } & \multicolumn{2}{c}{ Booth 1 } & \multicolumn{2}{c}{ Booth 2 } \\
& & $\lambda$ & $\mu$ & $\lambda$ & $\mu$ \\
\hline White & Black & 0.08 & 0.85 & 0.04 & 0.91 \\
White & Gray & 0.08 & 0.89 & 0.01 & 0.92 \\
Black & White & 0.1 & 0.9 & 0.07 & 0.86 \\
Black & Gray & 0.1 & 0.87 & 0.09 & 0.88 \\
Gray & White & 0.1 & 0.7 & 0.07 & 0.9 \\
Gray & Black & 0.03 & 0.95 & 0.03 & 0.92 \\
\hline
\end{tabular}

\section{An Example and Discussion}

5.1. An Example. The method put forward in previous sector has been used to evaluate paint quality at an automotive paint shop. Three types of double color cars are painted in one production line with two painting booths, which can be seen as a two-stage manufacturing system. The main color is always painted in the first painting booth and the minor color in the second. The color of the three double color cars is listed in Table 1.

Of course the quality transition probabilities from the different color differ. Based on historical data, transition probabilities $\lambda_{i j}$ and $\mu_{i j}$ are calculated. Table 2 lists the transition probabilities of the two booths. It has been shown that $\lambda_{i j}$ is usually small and $\mu_{i j}$ is large. Since the operation has no concern about quality performance of the system, it can be seen as the random organized production sequencing. Under that situation, numerical experiments are carried out to simulate the quality performance by randomly choosing the product sequence. Here we assume that the rate of incoming conforming car white body is identical. We obtain that the average quality ratio is only about 0.75 . There is only $0.57 \%$ difference compared with the actual data (the data has been changed because of the confidentiality).

Then, the transition data has been used to investigate the impact of production operation on quality performance of the system. Using the above method, an optimal sequence car 2 -car 1 -car 3 is obtained. The quality rate is 0.866 , and $15.9 \%$ quality improvement is achieved. Here although the improvement is not too large but it should be noted that this improvement is achieved only by changing the production sequence, which is almost with no cost.

There are two kinds of the product sequencing to produce the three types of cars in total. They are sequence car 1-car 2car 3 and car 1-car 3-car 2, respectively. Quality performances of the two sequences are $P\left(g_{b t}^{1}\right)=0.747$ and $P\left(g_{b t}^{2}\right)=0.866$, respectively.

It is easy to see that the product sequence car 1-car 3 -car 2 has a better quality performance than that of the sequence of car 1-car 2-car 3 even for batch policy and this point has been verified from the actual production data. We wanted to know why there is a big difference of the rate of conforming output product quality efficiency and this change is the result of different transaction state from batch to batch. The average stage quality efficiency is 0.817 and 0.921 for the sequence one and 0.91 and 0.961 for the second sequence, respectively. The second sequence has $11.4 \%$ and $4.3 \%$ higher stage efficiency than that of the first sequence because of the rate of the conforming input product which is identical for the two sequences. The second sequence has a smaller difference between the rate of the conforming input product and the stage efficiency which is the same as Corollary 2 showing that the second sequence should have a high rate of the conforming output product.

5.2. Discussion. In this research, we used the rate of conforming product to represent the incoming and outgoing quality and probability of system state as good or bad. The rate of conforming product is referred to as the probability of the good part. Another factor concerned for MMS is the cost; furthermore quality related cost should be involved in the model. For example, dissimilar part has different cost; optimal production operation should minimize the quality related cost defined as the quality related cost risk. This goal can be achieved by the following equation:

$$
\begin{aligned}
\min _{s_{i}^{l}} \quad R_{q} & =\sum_{i=1}^{n} \sum_{j=1}^{b_{s_{i}}} P\left(d_{s_{i}^{l}, j}\right) w_{s_{i}^{l}, j} \\
s_{i}^{l} & =1, \ldots, n, j=1, \ldots, b_{s_{i}^{l}}-1, k=1, \ldots, m .
\end{aligned}
$$

Here $R_{q}$ denotes the quality related cost risk and $w_{s_{i}^{l}, j}$ denotes the one part cost of the product type $i$ in $s_{i}^{l}$ sequence.

\section{Conclusion}

Quality performance is one of the most important aspects of the manufacturing systems. With the emergence of new manufacturing systems such as reconfiguration of a system and the dynamic and highly competitive marketplace demands, quality performance evaluation from multiaspect is becoming more and more vital. One of the aspects is to evaluate it from system level, namely, to predict quality performance from the rate of incoming conforming product to that of outgoing product in a statistical manner, where the detailed engineering knowledge along the multistage manufacturing system is not concerned. Based on the Markovian Chain model this paper approaches quality performance evaluation of multistage manufacturing system by considering the rate of incoming conforming product, production sequencing, and quality transition between batch to batch and quality propagation along the multistage. Different production policy is compared and optimal production sequencing is given. Two kinds of quality efficiency are put forward to facilitate the modeling and the discussion. Research shows optimal sequences should be such that the rate of incoming conforming product and the corresponding system quality transition have the same sequence, whereas batch size has 
minor influence on quality performance. The results of the research lead to the guidelines of quality management in multistage manufacturing systems. follows.

The future research works in this direction areas are as

(1) There could be situation that under bad machine state and defective incoming part stage can still produce a good part with small probability. Another considerable factor should be that under good machine state and good incoming part quality, there will be a small probability to produce bad quality part according to the stage process capability although the probabilities may be very small but those situations should be considered in the model.

(2) Configuration of manufacturing system has definitely a significant effect on system quality performance. It is needed to conduct research to unveil the relationship between manufacturing system configuration and quality performance, to know what kind of the configuration has a better performance than others.

(3) Only considering the rates of nonconforming units is not good enough for the research of multistage manufacturing system, which is one limitation of the proposal. Since the variation value of the quality character is more sensitive to the assignable causes of the system, the next study is to combine the variation value with the Markovian Chain to depict the quality performance of the manufacturing system.

\section{Appendix}

\section{A.}

From assumptions (7) (9), the $k$ th stage should be in normal state $o_{s_{i}^{l}, j, k}$ and abnormal state $f_{s_{i}^{l}, j, k}$ with the probability $P\left(o_{s_{i}, j, k}\right)$ and $P\left(f_{s_{i}^{l}, j, k}\right)$, and $P\left(f_{s_{i}^{l}, j, k}\right)+P\left(o_{s_{i}, j, k}\right)=1$ and also $P\left(f_{s_{i}^{l}, j-1, k}\right)+P\left(o_{s_{i}^{l}, j-1, k}\right)=1$.

So from (7) we have

$$
\begin{aligned}
P\left(o_{s_{i}^{l}, j, k}\right)= & P\left(o_{s_{i}^{l}, j-1, k}\right) \cdot\left(1-\lambda_{s_{i}^{l}, s_{i}^{l}, k}\right)+P\left(f_{s_{i}^{l}, j-1, k}\right) \\
& \cdot \mu_{s_{i}^{l}, s_{i}^{l}, k} \\
= & P\left(o_{s_{i}^{l}, j-1, k}\right) \cdot\left(1-\lambda_{s_{i}^{l}, s_{i}^{l}, k}\right) \\
& +\left(1-P\left(o_{s_{i}^{l}, j-1, k}\right)\right) \cdot \mu_{s_{i}^{l}, s_{i}^{l}, k}
\end{aligned}
$$

And from (5), we have

$$
P\left(o_{s_{i}^{l}, j-1, k}\right)=\frac{P\left(g_{s_{i}^{l}, j-1, k}\right)}{P\left(g_{s_{i}^{l}, j-1, k-1}\right)}=e_{s_{i}^{l}, j-1, k} .
$$

So we can get

$$
\begin{aligned}
P\left(o_{s_{i}^{l}, j, k}\right)= & P\left(o_{s_{i}^{l}, j-1, k}\right) \cdot\left(1-\lambda_{s_{i}^{l}, s_{i}^{l}, k}\right) \\
& +\left(1-P\left(o_{s_{i}^{l}, j-1, k}\right)\right) \cdot \mu_{s_{i}^{l}, l_{i}^{l}, k} \\
= & e_{s_{i}^{l}, j-1, k} \cdot\left(1-\lambda_{s_{i}^{l}, s_{i}^{l}, k}\right)+\left(1-e_{s_{i}^{l}, j-1, k}\right) \\
& \cdot \mu_{s_{i}^{l}, s_{i}^{l}, k} .
\end{aligned}
$$

Therefore from (3) we obtain

$$
\begin{aligned}
P\left(g_{s_{i}^{l}, j, k}\right) & \\
= & P\left(g_{s_{i}^{l}, j, k-1}\right) \\
& \cdot\left[e_{s_{i}^{l}, j-1, k}\left(1-\lambda_{s_{i}^{l}, s_{i}^{l}, k}\right)+\left(1-e_{s_{i}^{l}, j-1, k}\right) \mu_{s_{i}^{l}, s_{i}^{l}, k}\right] .
\end{aligned}
$$

B.

From the definition of quality efficiency, we need to verify the following equation:

$$
\begin{aligned}
e_{s_{i}^{l}, j, k}-e_{s_{i}^{l}, j-1, k} & =\delta_{s_{i}^{l}, s_{i}^{l}, k} e_{s_{i}^{l}, j-1, k}+\mu_{s_{i}^{l}, s_{i}^{l}, k}-e_{s_{i}^{l}, j-1, k} \\
& =\mu_{s_{i}^{l}, s_{i}^{l}, k}-\left(1-\delta_{s_{i}^{l}, s_{i}^{l}, k}\right) e_{s_{i}^{l}, j-1, k}>0 .
\end{aligned}
$$

Because $T_{s_{i}^{l}, s_{i}^{l}, k}=\mu_{s_{i}^{l}, s_{i}^{l}, k} /\left(\lambda_{s_{i}^{l}, s_{i}^{l}, k}+\mu_{s_{i}^{l}, s_{i}^{l}, k}\right)$ and $T_{s_{i}^{l}, s_{i}^{l}, k}>$ $e_{s_{i}^{l}, j-1, k}$

so $\mu_{s_{i}^{l}, s_{i}^{l}, k} /\left(\lambda_{s_{i}^{l}, s_{i}^{l}, k}+\mu_{s_{i}^{l}, s_{i}^{l}, k}\right)>e_{s_{i}^{l}, j-1, k}$; then we get $\mu_{s_{i}^{l}, s_{i}^{l}, k}>$ $\left(\lambda_{s_{i}^{l}, s_{i}^{l}, k}+\mu_{s_{i}^{l}, s_{i}^{l}, k}\right) e_{s_{i}^{l}, j-1, k}$.

From the definition of $\delta_{s_{i}^{l}, s_{i}^{l}, k}=1-\lambda_{s_{i}^{l}, s_{i}^{l}, k}-\mu_{s_{i}^{l}, s_{i}^{l}, k}$, we have $1-\delta_{s_{i}^{l}, s_{i}^{l}, k}=\lambda_{s_{i}^{l}, s_{i}^{l}, k}+\mu_{s_{i}^{l}, s_{i}^{l}, k}$.

And $\mu_{s_{i}^{l}, s_{i}^{l}, k}>\left(\lambda_{s_{i}^{l}, s_{i}^{l}, k}+\mu_{s_{i}^{l}, s_{i}^{l}, k}\right) e_{s_{i}^{l}, j-1, k}=\left(1-\delta_{s_{i}^{l}, s_{i}^{l}, k}\right) e_{s_{i}^{l}, j-1, k}$. Therefore $e_{s_{i}^{l}, j, k}>e_{s_{i}^{l}, j-1, k}$.

\section{Competing Interests}

The authors declare that they have no competing interests.

\section{Acknowledgments}

This research is supported by the National Natural Science Foundation of China (NSFC, Grant no. 51275049). The authors gratefully acknowledge the facilities provided by the Industrial Engineering Laboratory (IEL) at the Beijing Institute of Technology.

\section{References}

[1] R. R. Inman, D. E. Blumentfeld, N. Huang, and J. Li, "Production system design for quality: research opportunities from automotive industry prospective," International Journal of Production Research, vol. 4, pp. 1953-1971, 2003.

[2] D. E. Zoia, "Harbour outlines who's winning and why," September 2005, http://wardsauto.com.

[3] S. J. Hu, "Stream-of-variation theory for automotive body assembly," CIRP Annals-Manufacturing Technology, vol. 46, no. 1, pp. 1-6, 1997. 
[4] J. Jin and J. Shi, "State space modeling of sheet metal assembly for dimensional control," Journal of Manufacturing Science and Engineering, vol. 121, no. 4, pp. 756-762, 1999.

[5] D. Djurdjanovic and J. Ni, "Stream-of-variation (SoV)-based measurement scheme analysis in multistation machining systems," IEEE Transactions on Automation Science and Engineering, vol. 3, no. 4, pp. 407-422, 2006.

[6] Y. Ding, D. Ceglarek, and J. Shi, "Fault diagnosis of multistage manufacturing processes by using state space approach," Journal of Manufacturing Science and Engineering, Transactions of the ASME, vol. 124, no. 2, pp. 313-322, 2002.

[7] A. Khan, D. Ceglarek, and J. Ni, "Sensor location optimization for fault diagnosis in multi-fixture assembly systems," ASME Journal of Manufacturing Science and Engineering, vol. 120, no. 4, pp. 781-792, 1998.

[8] Y. Ding, D. Ceglarek, and J. Shi, "Modeling and diagnosis of multistage manufacturing processes, part I-state space model," in Proceedings of the Japan-USA Symposium, Ann Arbor, Mich, USA, 2000.

[9] S. M. Meerkov and L. Zhang, "Bernoulli production lines with quality-quantity coupling machines: monotonicity properties and bottlenecks," Annals of Operations Research, vol. 182, pp. 119-131, 2011.

[10] F. Ju, J. Li, G. Xiao, J. Arinez, and W. Deng, "Modeling, analysis, and improvement of integrated productivity and quality system in battery manufacturing," IIE Transactions, vol. 47, no. 12, pp. 1313-1328, 2015.

[11] F. Lolli, R. Gamberini, C. Giberti, M. Gamberi, M. Bortolini, and E. Bruini, "A learning model for the allocation of training hours in a multistage setting," International Journal of Production Research, vol. 54, no. 19, pp. 5697-5707, 2016.

[12] A. J. Lozano and A. L. Medaglia, "Scheduling of parallel machines with sequence-dependent batches and product incompatibilities in an automotive glass facility," Journal of Scheduling, vol. 17, no. 6, pp. 521-540, 2014.

[13] A. Elkamel, Scheduling of process operations using mathematical programming techniques: towards a prototypedecision support system [Ph.D. thesis], Purdue University, 1993.

[14] M. Fiedler, "On the limited potential of buffers to improve quality of experience," in Proceedings of the 2014 IEEE International Conference on Pervasive Computing and Communication Workshops (PERCOM WORKSHOPS '14), pp. 419-424, Budapest, Hungary, March 2014.

[15] H. Ishibuchi and T. Murata, "A multi-objective genetic local search algorithm and its application to flowshop scheduling," IEEE Transactions on Systems, Man and Cybernetics Part C, vol. 28, no. 3, pp. 392-403, 1998.

[16] A. Younes, A. Elkamel, M. Leung, C. Tzoganakis, and A. Lohi, "The quality-constrained scheduling problem in plastics compounding," Canadian Journal of Chemical Engineering, vol. 91, no. 7, pp. 1229-1243, 2013.

[17] M. Leung, Production scheduling optimization of a plastics compounding plant with quality constraints [M.S. thesis], University of Waterloo, Waterloo, Canada, 2009.

[18] Y. Koren, S. J. Hu, and T. W. Weber, "Impact of manufacturing system configuration on performance," CIRP AnnalsManufacturing Technology, vol. 47, no. 1, pp. 369-372, 1998.

[19] J. Li and N. Huang, "Quality evaluation in flexible manufacturing systems: a Markovian approach," Mathematical Problems in Engineering, vol. 2007, Article ID 57128, 24 pages, 2007.
[20] J. W. Wang, J. S. Li, J. Arinez, and S. Biller, "Product sequencing with respect to quality in flexible manufacturing systems with batch operations," IEEE Transactions on Automation Science and Engineering, vol. 7, no. 4, pp. 776-790, 2010.

[21] J. Wang, J. Li, J. Arinez, and S. Biller, "Improving quality in flexible manufacturing systems: a bottleneck transition approach," in Proceedings of the 6th Annual IEEE International Conference on Automation Science and Engineering (CASE '10), pp. 610-616, Toronto, Canada, August 2010.

[22] F. P. Zhang, J. J. Li, Y. Yan, J. P. Lu, and S. Y. Tang, "Quality evaluation of multistage manufacturing systems by jointly considering the incoming part quality and system conditions," in Proceedings of the ASME International Manufacturing Science and Engineering Conference (MSEC '13), Madison, Wis, USA, June 2013.

[23] R. Mantripragada and D. E. Whitney, "Modeling and controlling variation propagation in mechanical assemblies using state transition models," IEEE Transactions on Robotics and Automation, vol. 15, no. 1, pp. 124-140, 1999.

[24] C. Gagné, M. Gravel, and W. L. Price, "Solving real car sequencing problems with ant colony optimization," European Journal of Operational Research, vol. 174, no. 3, pp. 1427-1448, 2006.

[25] M. Prandtstetter and G. R. Raidl, "An integer linear programming approach and a hybrid variable neighborhood search for the car sequencing problem," European Journal of Operational Research, vol. 191, no. 3, pp. 1004-1022, 2008. 


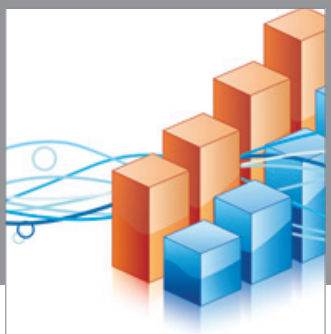

Advances in

Operations Research

vatem alat4

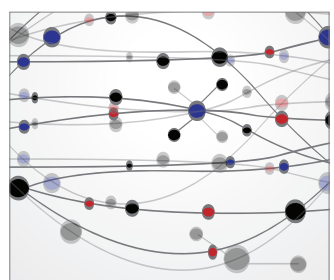

\section{The Scientific} World Journal
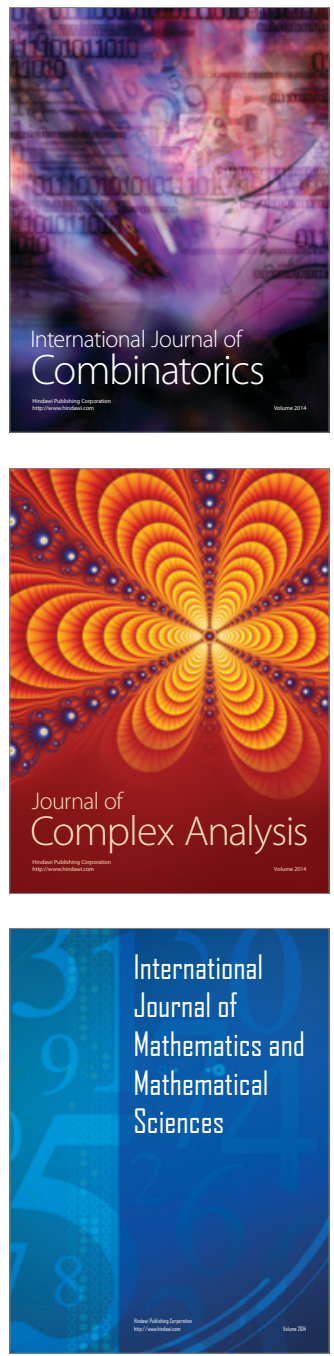
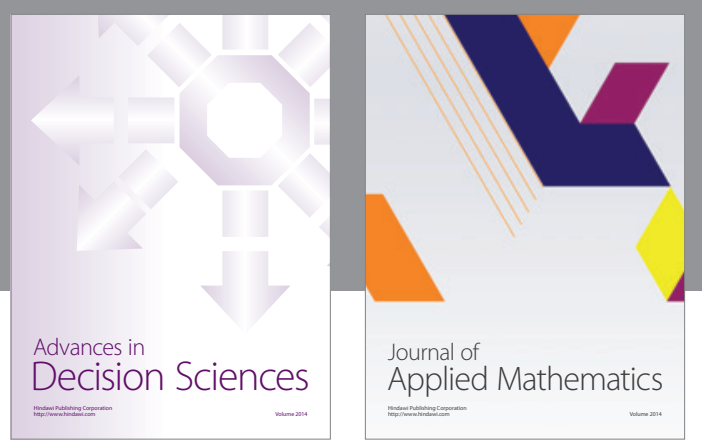

Algebra

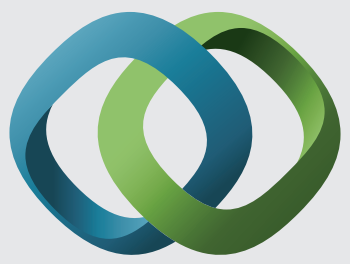

\section{Hindawi}

Submit your manuscripts at

http://www.hindawi.com
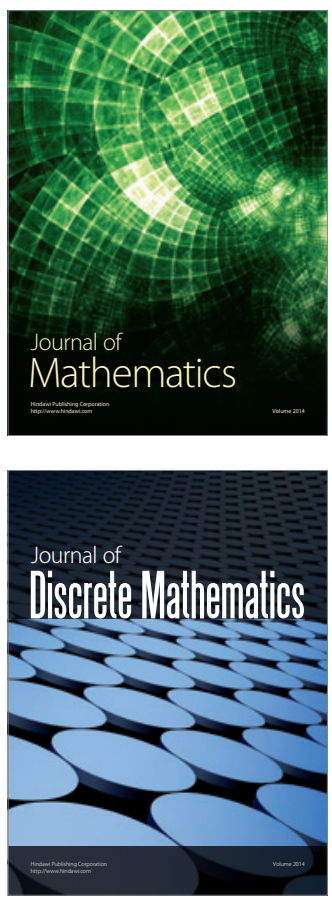

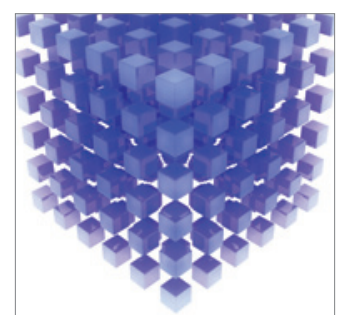

Mathematical Problems in Engineering
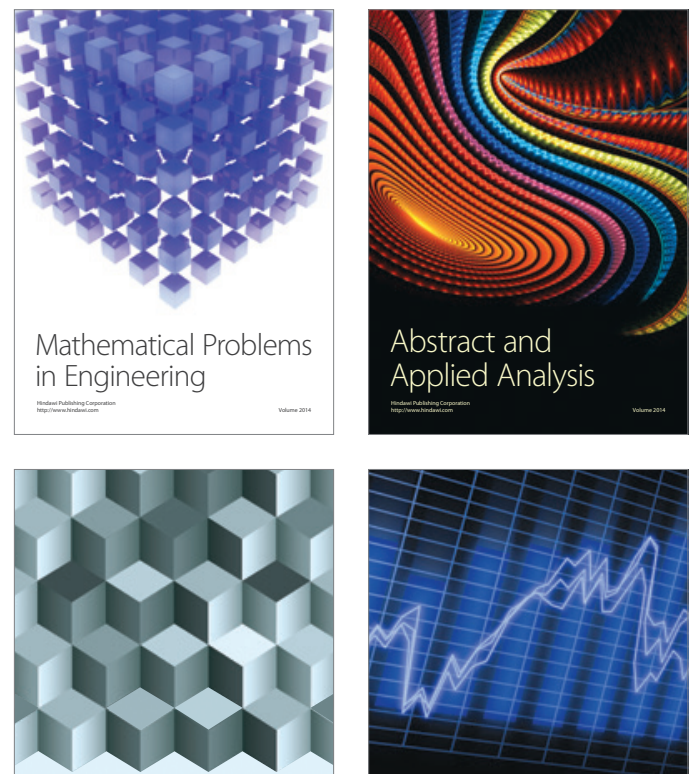

Journal of

Function Spaces

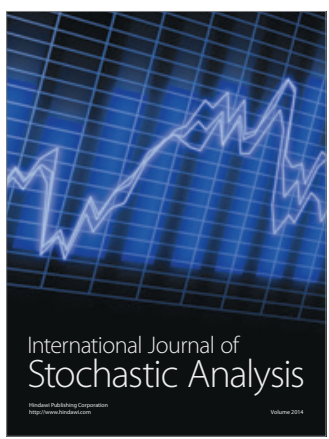

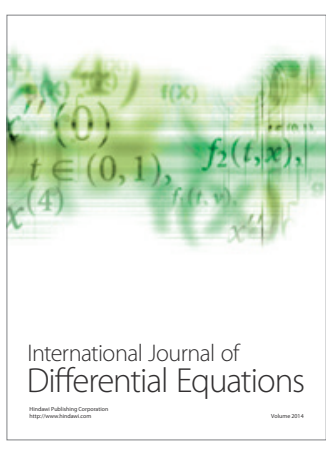
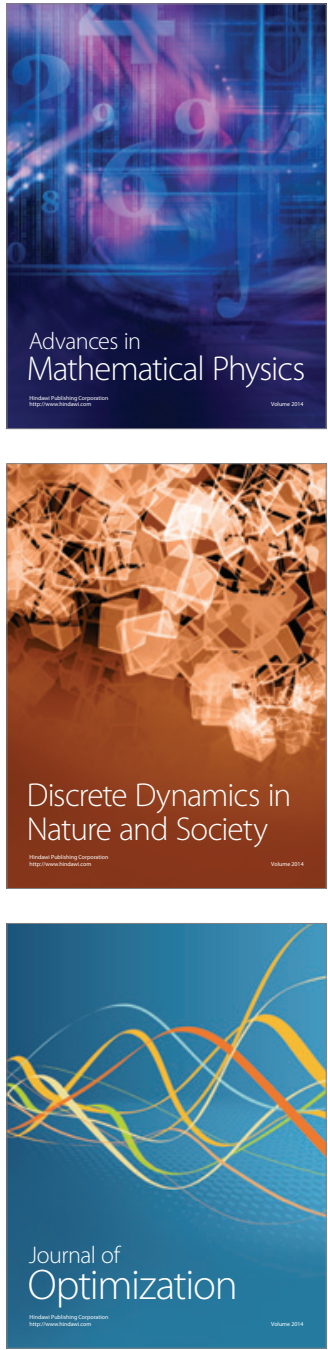